\title{
Web Crawler and Web Crawler Algorithms: A Perspective
}

\author{
K Velkumar, P Thendral
}

\begin{abstract}
A web crawler is also called spider. For the intention of web indexing it automatically searches on the $W W W$. As the W3 is increasing day by day, globally the number of web pages grown massively. To make the search sociable for users, searching engine are mandatory. So to discover the particular data from the $W W W$ search engines are operated. It would be almost challenging for mankind devoid of search engines to find anything from the web unless and until he identifies a particular URL address. A central depository of HTML documents in indexed form is sustained by every search Engine. Every time an operator gives the inquiry, searching is done at the database of indexed web pages. The size of a database of every search engine depends on the existing page on the internet. So to increase the proficiency of search engines, it is permitted to store only the most relevant and significant pages in the database.
\end{abstract}

Keywords: Web Crawler, Focused Crawler, Web crawler algorithms

\section{INTRODUCTION}

WWW is the most generally known and significant source of information for data mining research. So, it becomes a challenging task to retrieve useful and novel information and knowledge from this huge, dynamic, structurally complex and ever-growing World Wide Web. Webmine is a process of Datamining methods to routinely ascertain and mining the information from website files and amenities. The prime motive of webmining is to ascertain beneficial data on the WWW and its custom patterns. Webmining is broadly classified into three kinds of mining techniques namely: Webcontent (WCM), Webstructure (WSM) and webusage mining (WUM). Content mining retrieve knowledge from its content from web documents. Web structure mining retrieve the configurative data from the internet. Web usage mining identifies or explores interesting usage patterns to huge quantity of information [15].

In current trend web pages are increasing day by day. We need search engine to retrieve the data from world wide web repository. The search engines are software that explore data

Revised Manuscript Received on May 15, 2020.

* Correspondence Author

K Velkumar*, Assistant Professor, Department of Computer Science and Engineering, Theni Kammavar Sangam College of Technology, Theni, India, velkumar@klu.ac.in

P Thendral, Associate Professor, Department of Computer Science and Engineering, Kalasalingam Academy of Research and Education, Krishnankovil, thendralniti@gmail.com

(c) The Authors. Published by Blue Eyes Intelligence Engineering and Sciences Publication (BEIESP). This is an open access article under the CC BY-NC-ND license (http://creativecommons.org/licenses/by-nc-nd/4.0/) from the website. The web crawling algorithm support the search engine to collect webpage from the internet. It occurs owing to the difficulty to retrieve the enormous data stored in internet. The searching engine rely on crawlers for identifying the related pages from the internet.

\section{Web CraWler}

Web crawler is a package that scans from the pages of internet and discover the information from web [1].

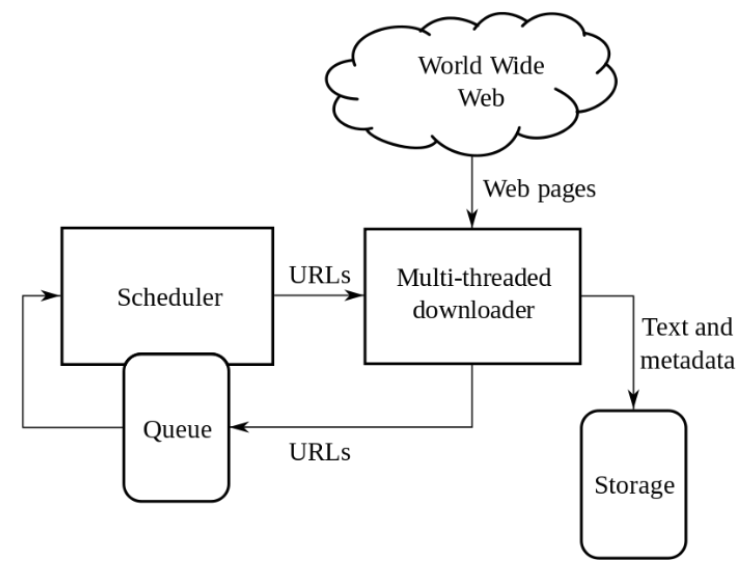

Figure 1 WC Architecture

Motivation for studying WC [2]

- A WC is a search engines retrieve the facts which is indexed by searching engine. The research identifies several limitations aswellas policies on swarming internet.

- The researcher attempts to discover several methods applied for network crawling to regulate the relative investigation. It also analyzes several subject scheme and performance measures pertained to various researches.

- The researcher commenced to work from the WC, as the absence of whole systematic literature survey as a stimulating factor. He investigated the entire repository for WC and précised it to report researcher for further study.

\section{Forms of web crawler}

Universal Crawler: This WC is insufficient to network pages of a specific content area. They save on the links which are endless and retrieve the whole pages they come across [3].

Preferential crawler: The preferential WC doesn't scan every links they confront rather one proposes a state of importance that control privileged crawling [4].

Hidden Web crawler: The volume of data from the website can't be retrieved straight succeeding hyperlinks on pages. The retrieved data are concealed under discovery or demand interface; these

Published By:

Blue Eyes Intelligence Engineering

\& Sciences Publication

(C) Copyright: All rights reserved.

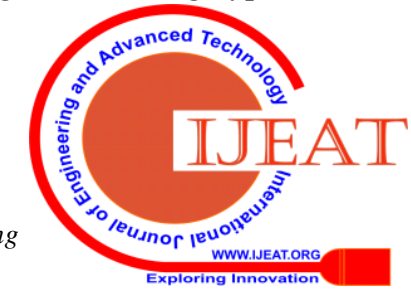




\section{Web Crawler and Web Crawler Algorithms: A Perspective}

section of the network page is called Hidden-web [5].

Mobile crawler: This type of mobile crawler is a collection and purification of web data. It is completed by the server itself [6].

Incremental crawler: Data given on the webpage is vital and tend to change often. It sustains the index repository of the search engine [7].

\section{Focused Crawler}

The focused crawler concentrates on the approaches that calculate whether a website page is linked to a specified set of areas in advance relocating it to a local repository. So it is called as a topical crawler. This method is divided into two classes: content-based and link-based [8].

The content based classification retrieves the information from the web as text mining. The keyword discovery method assists the bounds whether a web page's information confines the exact topic. The approaches of the method typically test the words on a website page and intersects a lists of exact method. The link-based method manipulates the link configuration from the webpage to comprehend data on the website pages. Anchor text is a term or expression which hyperlinks with a target webpage, known to be a valuable estimation as it comprises the depiction of the aimed page [9].

\section{Self-Adaptive Semantic Focused Crawler}

A SFC is a software that is intelligent enough to discover website, and download associated network data on exact subjects along with semantic technologies [17] [18]. The semantic advances give mutual information to refine the interoperability between heterogeneous parts, semantic invention was lengthily connected in the area from modern computerization. This intention of SFC is to decisively as well as to effectively recover also to copy important network data.

\section{Web CraWlers Algorithms}

There are many web crawler algorithms to extract useful knowledge from the website then to remove unwanted data.

The basic steps are implemented through WC algorithms and grasp a list of seed Uniform Resource Locators as its $\mathrm{i} / \mathrm{p}$ frequently accomplishes resulting stage [12]:

- To delete a Uniform Resource Locator from its list.

- The corresponding pages are downloaded

- Related page has to be checked.

- To discover any link which it contains.

- The URL list is added again with the link

- Afterward every URL are linked and the most significant pages are resumed.

Saranya[1] et al discussed five algorithms, BreadthFirst, BestFirst, FishSearch Algorithm, SharkSearch Algorithm, and PageRank Algorithm. Important factors like: accuracy, memory and F-score values the proficiency of the CA.

BreadthFirst algorithm is the easiest algorithm in finding data in the order of FIFO. BFS technique utilizes the boundary Uniform Resource Locator list for surfing the network pages. Frontier is the used Queue besides crawling the network in accordance to which they are confronted. To figure out page scores algorithms sort website pages by two kinds. When a website page supplies redundant data for particular domain, then that is characterized as authority website page. The Website page that impart network to authorization page are known as system. Weight is allocated to all system, as a result authorization pages, pagescore is summed consequently [11].

The BestFirst Search (BFS) is an experimental searching algorithm. In BFS method, appropriate summing is prepared for every network and the significant network, like one with the maximum relevant value, (i.e) to retrieve from the queue [13]. Hence always the finest accessible sources are surfed then utilized.

Fishsearch(FS) is a vibrant experimental searching algorithms. The FS functions on impulse which relate the links that have related neighbours; so it begins by means of a related network and delves indepth into the link then stop surfing from the link that is inappropriate. This main scope of FS algorithms relies on the maintenance of Uniform Research Locator [16].

Shark page algorithm, uses the equivalent simple metaphor and it leads to the retrieve of more equivalent data in the same examination period. This algorithm used relevant or irrelevant calculation of document application in order to sum up the relevant document to a given query [14].

The PageRank algorithm yields a possible sharing customized to characterize the probability which a user clicks on link to reach the specific page. The Page Rank is counted for the collection of file size. PR is expected from many researchers that the distribution is evenly distributed. The PR calculation requires passes, called "repetition", to adjust with the calculated collection PR values to ideally reflect the true values [1]. Finally, she proposed that the best characteristics from PageRank algorithm and BestFirst algorithm are jointly composed to deliver the best outputs [1].

Pradeep Sahoo et al proposed NDC (Noisy data cleaner algorithm) and Uniform Research Locator pattern extractor algorithm (UPE). UPE algorithm discovers all related data on the worker's request. The related data is the essential data on the web page that they need to look at [10]. NDC is very well-organized in eliminating unrelated data on the discovered webpages. The advantage of the Noisy-Data-Cleaner algorithms are efficient for huge amount of information also mini datasets. The NDC algorithms skillfully eliminates all types of noises. Results and performance Calculation

\section{RESULTS AND PERFORMANCE CALCULATION}

We analyzed the following algorithms such as web crawler algorithms, BreathFirst Algorithms, BestFirst Algorithms, FirstSearch, FishSearch, Shark-Search and Page -Rank algorithm. The CA algorithms provided the best performance of crawler. This comprised URL pattern extractor and noisy cleaner method which had the maximum exactness of 94 . \% for web page and $90.5 \%$ for news data set and $87.0 \%$ for image dataset [10]. The performance of crawler's algorithms important factors like: accuracy, memory and F-score values the proficiency of the CA.

Published By: (lue Eyes Intelligence Engineering \& Sciences Publication

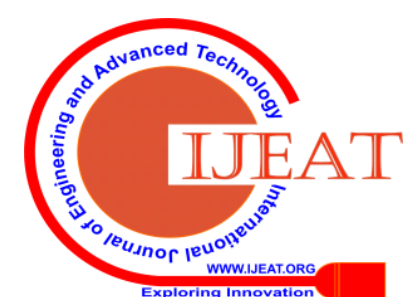




\section{CONCLUSION}

This paper reviews various web mining techniques, web crawler, Focused-Crawler, SASF Crawler and web crawling algorithms. In the web crawling algorithms BreathFirst Algorithms, BestFirst Algorithms, FirstSearch, FishSearch, Shark-Search and Page -Rank algorithm are reviewed.

\section{REFERENCES}

1. S. Saranya, B.S.E. Zoraida and P. Victor Paul, "A Study on Competent Crawling Algorithm (CCA) for Web Search to Enhance Efficiency of Information Retrieval", Artificial Intelligence and Evolutionary Algorithms in Engineering Systems, Advances in Intelligent Systems and Computing 325, DOI 10.1007/978-81-322-2135-7_2.

2. Manish Kumar, Rajesh Bhatia and Dhavleesh Rattan, "A survey of Web crawlers for information retrieval", WIREs Data Mining Knowl Discov 2017, e1218. doi: 10.1002/widm.1218.

3. Chakrabarti S, Van Den Berg M, Dom B. Focused crawling: a new approach to topic-specific Web resource discovery. Comput Networks 1999, 31:1623-1640. https://doi.org/10.1016/S1389-1286 (99)00052-3.

4. Yu HL, Bingwu L, Fang Y. Similarity computation of web pages of focused crawler. In: 2010 International Forum on Information Technology and Applications, 2010, 2, 70-72. https://doi.org/10.1109/IFITA.2010. 308.

5. 5. Gravano L, Ipeirotis PG, Sahami M. QProber: a system for automatic classification of hidden-web databases. ACM Trans Information Systems, 2003, 21:1-41. https:// doi.org/10.1145/635484.635485.

6. Hammer J, Fiedler J. Using mobile crawlers to search the Web efficiently. International of Journal of Computer Information Science, 2000, 1:36-58.

7. Badawi M, Mohamed A, Hussein A, Gheith M. "Maintaining the search engine freshness using mobile agent". Egypt Informatics J 2013, 14:27-36. https://doi.org/10.1016/j.eij.2012.11.001.

8. Chau M, Chen H (2003) "Comparison of three vertical search spiders". IEEE Computing, 36(5):56-62.

9. Jae-Gil Lee, Donghwan Bae, Sansung Kim1, Jungeun Kim1 and Mun Yong Yi, "An effective approach to enhancing a focused crawler using Google", The Journal of Supercomputing, https://doi.org/10.1007/s11227-019-02787-9.

10. Pradeep Sahoo and Rajagopalan Parthasarthy, "An Efficient Web Search Engine for Noisy Free Information Retrieval", The International Arab Journal of Information Technology, Vol. 15, No. 3, May 2018.

11. S. Jaiganesh, P. Babu, K. Nimmati Satheesh, Comparative study of various web search algorithms for the improvement of web crawler. Int J. Eng. Res. Technol. (IJERT) 2(4) (2013).

12. Menczer, Filippo, Gautam Pant, and Padmini Srinivasan, "Topical webcrawlers: Evaluating adaptive algorithms," ACM Transactions on Internet Technology (TOIT), vol. 4, no. 4, pp. 378-419, 2004.

13. Best First Search, Accessed March 14, 2013 , en.wikipedia.org/wiki/Best-first_search.

14. Jay Prakash and Rakesh Kumar,"Web Crawling through Shark-Search using PageRank", International Conference on Computer, Communication and Convergence (ICCC 2015) doi 10.1016/j.procs.2015.04.172

15. Anurag Kumar and Ravi Kumar Singh, "Web Mining Overview, Techniques, Tools and Applications: A Survey," International Research Journal of Engineering and Technology (IRJET), vol. 03, no. 12, pp. 1543-1547, December 2016.

16. Aviral Nigam, "Web Crawling Algorithms", International Journal of Computer Science and Artificial Intelligence, Sept. 2014, Vol. 4 Iss. 3, PP. 63-67.

17. Farookh Khadeer Hussain Hai Dong, "Self-Adaptive Semantic Focused Crawler for Mining Services Information Discovery," INDUSTRIAL INFORMATICS, vol. 10, pp. 1616-1626, MAY 2014.

18. J. L. M. Lastra and M. Delamer, "Semantic web services in factory automation: Fundamental insights and research roadmap," IEEE Trans. Ind. Informat, vol. 6, no. 1, pp. 1-11, Feb 2006.

19. Prakash, Jay, and Rakesh Kumar. "Web Crawling through Shark-Search using PageRank", Procedia Computer Science, 2015.
20. Liu, Shengjian, and Xiaoning Wu. "Architecture design of IT education platform based on web mining", 2011 IEEE International Conference on Computer Science and Automation Engineering, 2011.

21. Hsinchun Chen. "Introduction to theJASIST Special Topic issue on web retrieval and mining: A machine learning perspective", Journal of the American Society for Information Science and Technology, 2003.

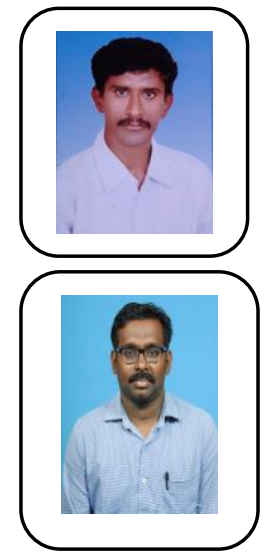

\section{AUTHORS PROFILE}

K Velkumar, completed B.Tech (IT), ME (CSE), has been working as an Assistant Professor in Then Kammavar Sangam College of Technology for the past 13 years. His research area is Web mining.

Dr. P Therndral, completed BE(ECE), ME(SS) and $\mathrm{PhD}(\mathrm{CSE})$, has been working as an Associate Professor in Kalasalingam Academy of Research and Education. His research area is Computational Intelligence and Algorithm Design.

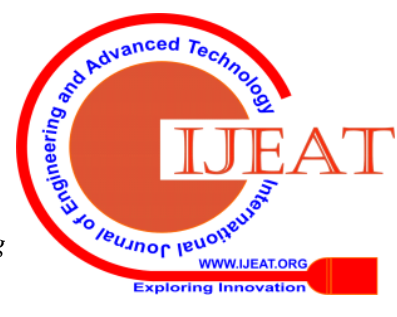

